\title{
Talaromyces marneffei and Mycobacterium tuberculosis co-infection in a patient with high titer anti-interferon- $\gamma$ autoantibodies: a case report
}

\author{
Ye Qiu ${ }^{1,2}$, Mianluan Pan ${ }^{1}$, Zhenming Yang ${ }^{3}$, Wen Zeng ${ }^{3}$, Hui Zhang ${ }^{3}$, Zhengtu Li ${ }^{4}$ and Jianquan Zhang ${ }^{\text {* }^{*}}$ (1)
}

\begin{abstract}
Background: High-titer anti-interferon (IFN)- $\gamma$ autoantibodies are strongly associated with intracellular pathogens such as nontuberculous mycobacteria and Talaromyces marneffei, but they are not as commonly associated with Talaromyces marneffei co-infected with Mycobacterium tuberculosis.

Case presentation: Herein, we report a case of an HIV-negative Chinese man with a severe, disseminated co-infection of Talaromyces marneffei and Mycobacterium tuberculosis, who had a high-titer of anti IFN- $y$ autoantibodies and a CFI heterozygous nonsense gene mutation. The patient rapidly developed sepsis and died. Through by flow cytometry for CD4 ${ }^{+}$T cells' intracellular phosphorylated STAT-1 and Th1 cells (CD4 ${ }^{+}$IFN- $\gamma^{+}$cells), we found that the patient's serum can inhibited IFN $\gamma^{\text {-induced }} \mathrm{CD} 4^{+} \mathrm{T}$ cells'STAT-1 phosphorylation and Th1 cell differentiation in normal peripheral blood mononuclear cells, but this phenomenon was not observed in normal control's serum. In addition, the higher serum concentration in the culture medium, the more obvious inhibition of Th1 cell differentiation.

Conclusions: For HIV-negative individuals with relapsing, refractory, fatal double or multiple intracellular pathogen infections, especially Talaromyces marneffei, clinicians should be aware that if they might be dealing with adult-onset immunodeficiency syndrome due to high-titer anti-IFN- $\gamma$ autoantibodies. Systematic genetic and immunological investigations should also be performed.
\end{abstract}

Keywords: Talaromyces marneffei, Mycobacterium tuberculosis, Anti-interferon-gamma autoantibody, HIV-negative patient, Case report

\section{Background}

Adult-onset immunodeficiency syndrome due to hightiter anti-interferon (IFN) $-\gamma$ autoantibodies is considered to be a susceptibility factor for intracellular pathogens infection, especially nontuberculous mycobacteria and Talaromyces marneffei in Southeast Asia [1-3]. However, the specific mechanism of immune deficiency of

\footnotetext{
*Correspondence: jqzhang2002@sina.com

1 Department of Respiratory and Critical Medicine, The Eighth Affiliated Hospital, Sun Yat-Sen University, Shenzhen 518000, Guangdong, China Full list of author information is available at the end of the article
}

anti-IFN- $\gamma$ autoantibodies is still unclear. In addition, anti-IFN- $\gamma$ autoantibodies have not widely been $\operatorname{cog}$ nizant in tuberculosis [4]. Thus, we report a case of a 56-year-old HIV-negative Chinese man, with a high levels of anti-IFN- $\gamma$ autoantibodies, simultaneously diagnosed disseminated Mycobacterium tuberculosis and Talaromyces marneffei co-infection by using metagenomics nextgeneration sequencing (mNGS), aims to attract clinical attention and attempts to study the possible immune deficiency mechanism of anti-IFN- $\gamma$ autoantibodies. 


\section{Case presentation}

A 56-year-old Chinese man with coronary atherosclerotic heart disease was admitted to the local hospital on June 22, 2019 for a 4-month period due to expectoration, fever (body temperature: $39-40{ }^{\circ} \mathrm{C}$ ), weight loss, and multiple lymphadenopathy. The patient also had significantly increased white blood cell (WBC) and neutrophil (N) counts as well as an increased erythrocyte sedimentation rate (ESR) and C-reactive protein (CRP) and procalcitonin (PCT) levels (Fig. 1A). He was nonresponsive to intermittent antibacterial therapy for 2 months (comprising cefoperazone sulbactam and moxifloxacin), and his condition deteriorated due to neck, armpit, and groin lymphadenopathy, jaundice, and proteinuria. Lymphocyte subset counts and percentages were normal. The patient had low levels of circulating factor I at $43 \%$ (normal value: $70-120 \%$ ), but normal complement C3 and $\mathrm{C} 4$ levels. Thrombocytopenia, jaundice, and acute renal failure rapidly manifested later (Fig. 1A). HIV, antinuclear antibody, and anti-double stranded DNA tests were all negative. Chest computerized tomography $(\mathrm{CT})$ revealed bilateral pulmonary infiltration with mediastinal lymphadenopathy, multiple bone destruction, and pleural and pericardial effusions (Fig. 1B). The emission CT showed a significantly increased uptake in multiple bones (Fig. 1C). A yellowish pleural effusion and its exudative manifestations and a marked increase in protein content and predominantly high levels of neutrophils (85\%) were observed. The concentration of pleural fluid adenosine deaminase was $3.4 \mathrm{U} / \mathrm{L}$. Histopathology of the lymph node and pulmonary lesions revealed granulomatous inflammation. Furthermore, no evidence of organisms or malignancy was identified in the bronchoscopy alveolar lavage fluid (BALF), blood, pleural effusion, bone marrow, lymphatic, or lung tissues when tested using microbial smears (negative acid-fast bacilli), cultures, and pathological examinations. However, Mycobacterium tuberculosis (TB) and Talaromyces marneffei were identified using Metagenomic next-generation sequencing (mNGS) [5] from the bronchoscopy analysis of the alveolar lavage fluid and cervical lymph nodes. The sputum and bone marrow were also analyzed for pathogen cultures using mNGS. Following a 3-day regimen of anti-tuberculosis treatment and antifungal therapy
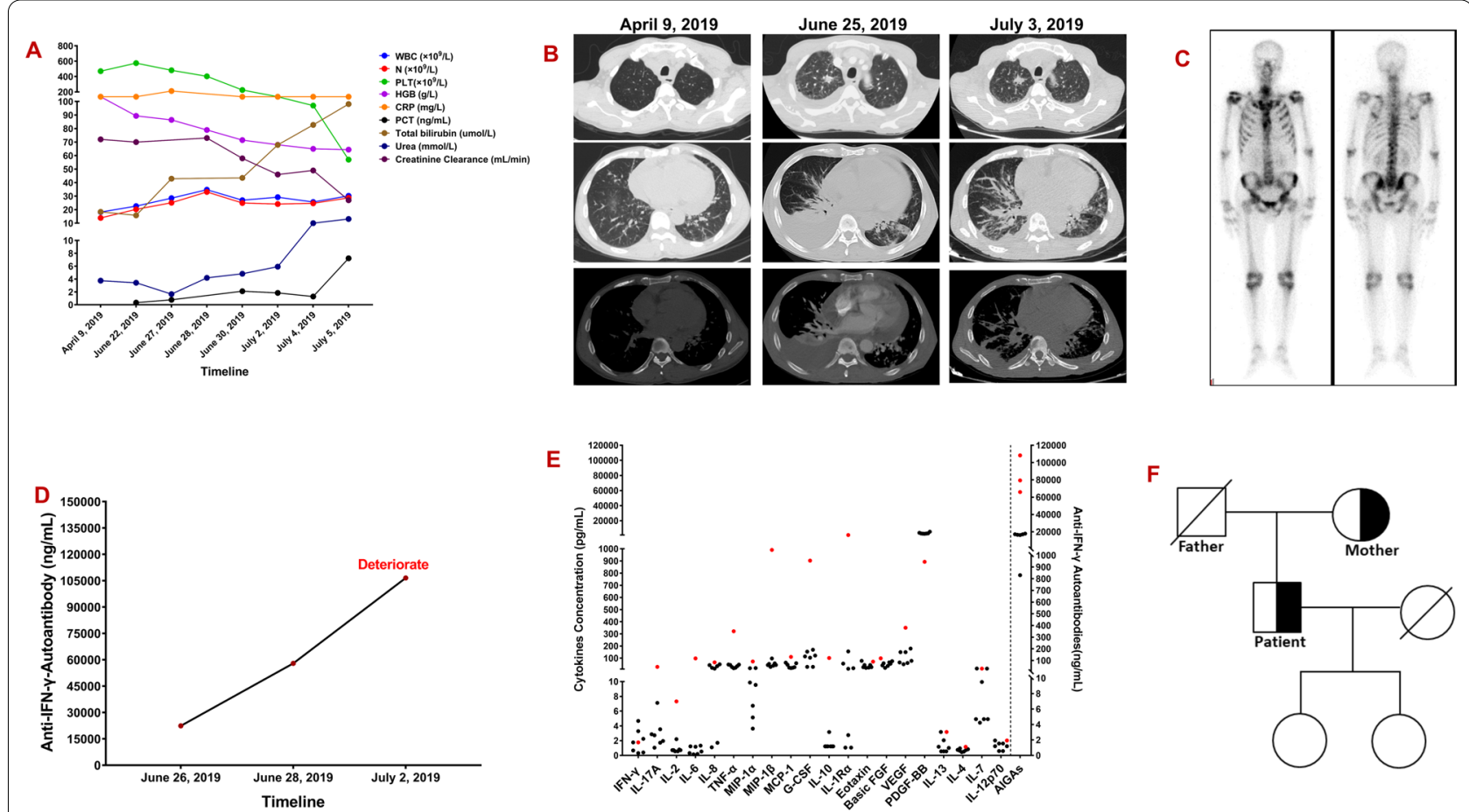

Fig. 1 A Inflammatory markers, liver, and kidney function: white blood cell (WBC) and neutrophil (N) counts, and C-reactive protein (CRP), procalcitonin (PCT), urea and total bilirubin levels rapidly increased; platelets (PLT), hemoglobin (HGB) level, and creatinine clearance decreased rapidly. B Computed tomography dynamic monitoring series: pulmonary lesions, pleural effusions, pericardial effusions and osteolysis. C Emission computed tomography: significantly increased uptake in multiple ribs and vertebrae, left sacroiliac spine, and left acetabulum. D The patient's anti-IFN- $\gamma$ autoantibody titer increased significantly as the condition worsened during the disease course. E Multiplex screening of serum from the patient and 7 normal control plasmas for cytokines and anti-IFN- $\gamma$ autoantibodies. F Pedigree tree. Whole-genome sequencing of the proband (patient) and his mother revealed a heterozygous nonsense mutation (c.559 C>T; p. Arg187*) in CFI 
(amphotericin B liposome combined with voriconazole), the patient died of septic shock. On the 3rd day after his death, Talaromyces marneffe $i$ was isolated from the sputum and Mycobacterium tuberculosis was identified in the bone marrow using mNGS.

The patient, his mother, his two healthy daughters, and seven healthy controls, were recruited from the First Affiliated Hospital of Guangxi Medical University between June 2019 and July 2019. All subjects provided written informed consent. This study was approved by the Ethical Review Committee of the First Affiliated Hospital of Guangxi Medical University (2020.KY-E032). Detailed methods for Anti-IFN- $\gamma$ autoantibody assay, Bio-Plex ${ }^{\mathrm{TM}} 25$ cytokine assay, flow cytometry, DNA extraction, DNA libraries and sequencing, and bioinformatics analysis are provided in Additional file 1.
The patient's serum showed a remarkably high-titer of anti-IFN- $\gamma$ autoantibody, and the titer increased significantly as the condition worsened during the course of the disease. The patient's serum IL-17 A, IL-2, IL-6, IL-8, TNF- $\alpha$, MIP- $1 \alpha$, MIP- $1 \beta$, MCP-1, G-CSF, IL-10, and IL-1R $\alpha$ levels were also increased significantly than the seven healthy controls' (Fig. 1D). However, the IFN- $\gamma$ levels were in the same range as those in the healthy controls. Whole exome sequencing (WES) of the proband and his mother revealed a heterozygous CFI nonsense mutation (c.559 C>T; p. Arg187*; Figs. 1F and 2). The CFI gene (CFI, OMIM*217030) comprises 13 exons localized on chromosome 4q25 and its deficiency is inherited in an autosomal recessive manner. WES verification revealed that the patient's mother was also a carrier of the heterozygous variant.

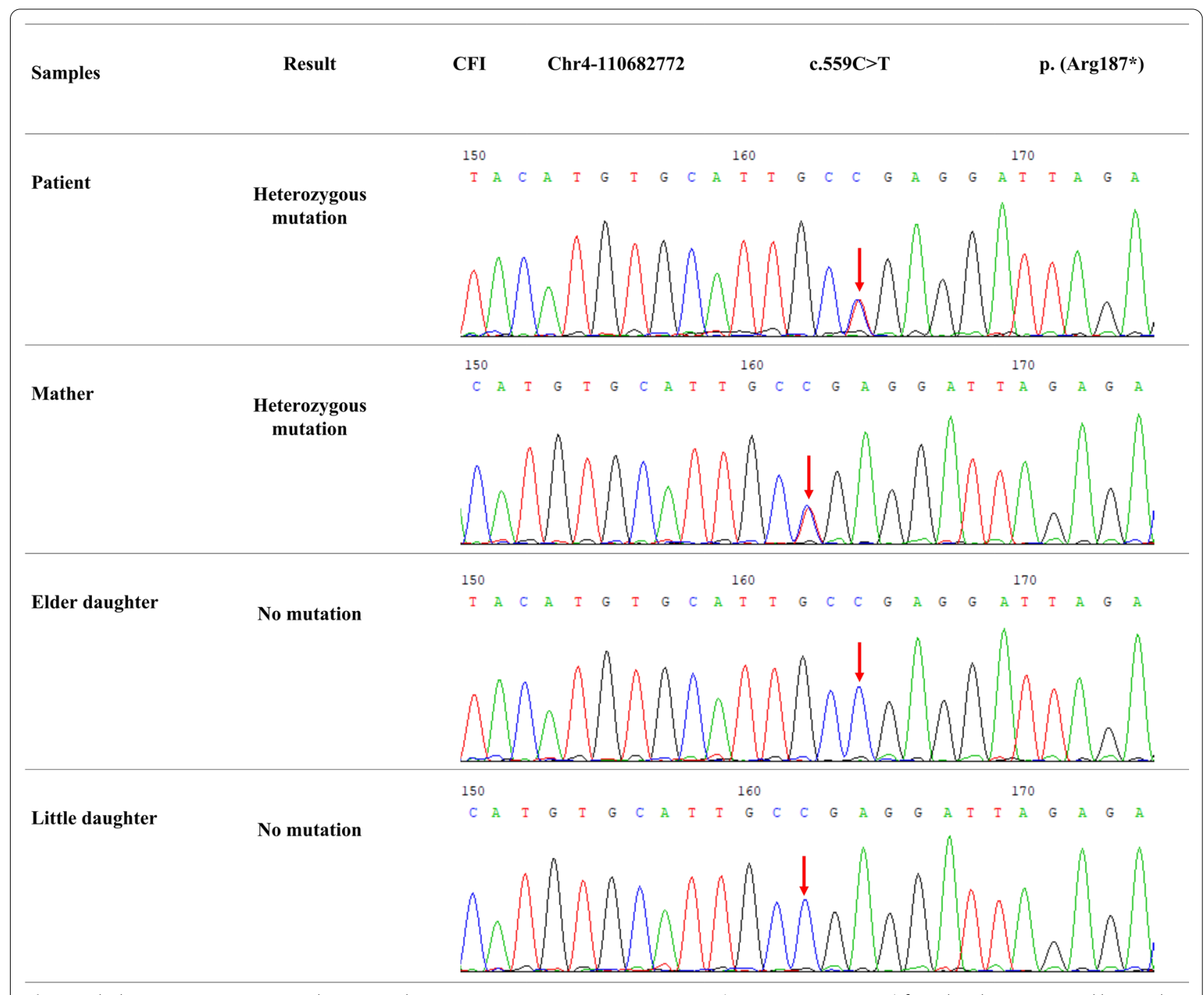

Fig. 2 Whole-exome sequencing indicate a CFI heterozygous nonsense gene mutation (c.559 C>T; p. Arg187*) found in the patient and his mother 
Through by flow cytometry for $\mathrm{CD} 4^{+} \mathrm{T}$ cells' intracellular phosphorylated STAT-1 and Th1 cells $\left(\mathrm{CD} 4^{+}\right.$ IFN- $\gamma^{+}$cells), we found that the patient's serum can remarkably inhibited IFN $\gamma$-induced $\mathrm{CD}^{+}{ }^{+} \mathrm{T}$ cells' STAT-1 phosphorylation and Th1 cell differentiation in normal peripheral blood mononuclear cells, but this phenomenon was not observed in normal control's serum. However, when the patient PBMCs were washed free of the patient's serum, they demonstrated normal IFN $\gamma$-induced STAT-1 phosphorylation in $\mathrm{CD}^{+}{ }^{+} \mathrm{T}$ cells and Th1 cell differentiation (Fig. 3). In addition, the higher serum concentration in the culture medium, the more obvious inhibition of Th1 cell differentiation.

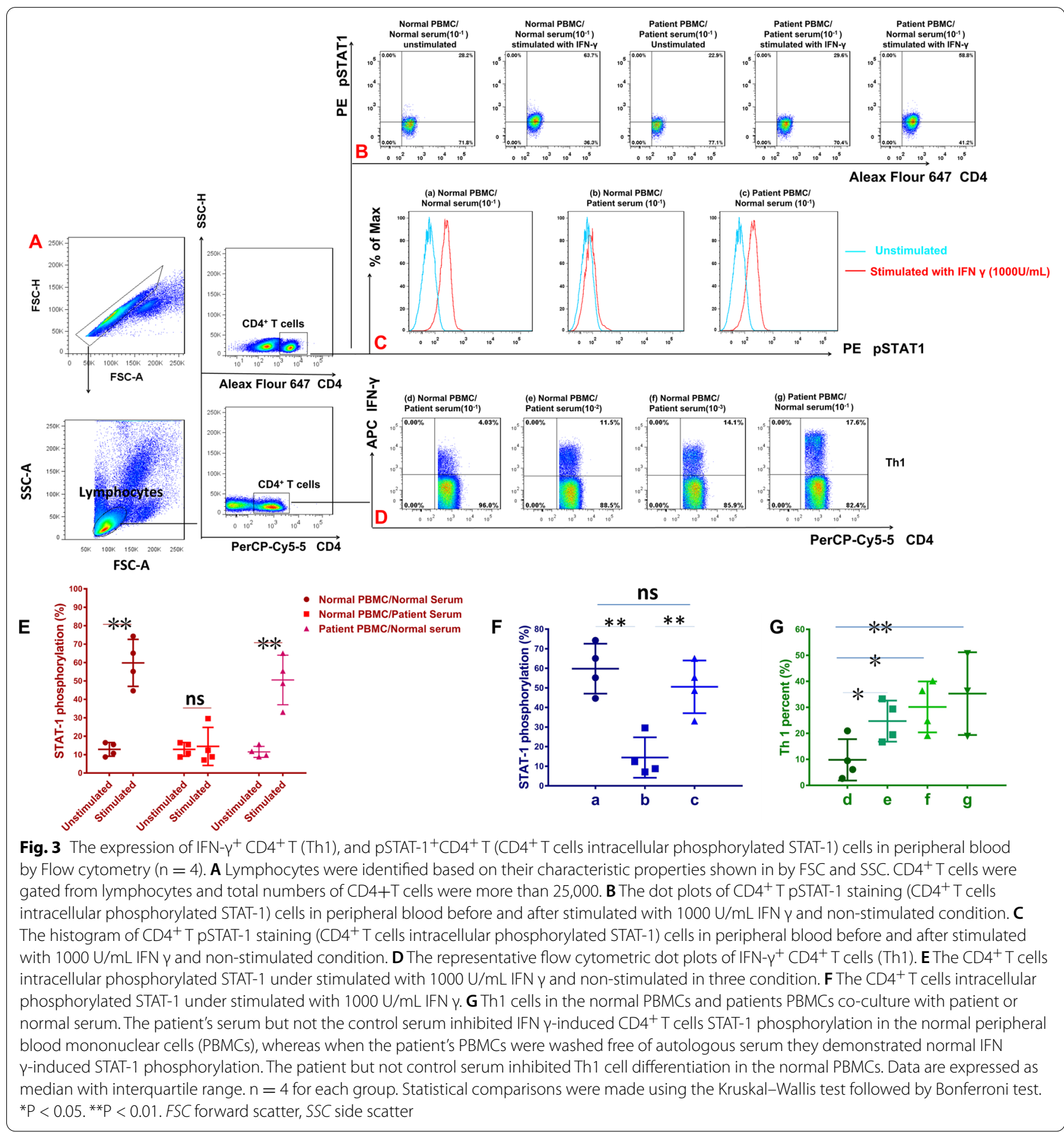




\section{Discussion and conclusion}

A high-titer of anti-IFN- $\gamma$ autoantibodies is strongly associated with intracellular pathogens especially in Nontuberculous mycobacteria and Talaromyces marnef$f e i[1]$. Cao et al. found that there was a high prevalence of neutralizing anti-IFN- $\gamma$ autoantibodies (94.8\%) in 58 HIV-negative adults with severe Talaromyces marneffei infections who were otherwise healthy [5]. In our previously researches, we found that it showed that adultonset immunodeficiency syndrome, due to high-titer of anti-IFN- $\gamma$ autoantibodies were the most common underlying immunodeficiency in HIV-negative Talaromyces marneffei infection patients [6]. In addition, the ratio of actual value to cut-off value of anti-IFN- $\gamma$ autoantibodies was an independent risk factor of TM infection [2]. However, the immune deficiency mechanism of antiIFN- $\gamma$ autoantibodies in Talaromyces marneffe $i$ infection is less clear.

Lightly increased levels of anti-IFN- $\gamma$ autoantibodies were found in patients with severe pulmonary tuberculosis [1]. However, the neutralization or biological activity of lightly increased levels of anti-IFN- $\gamma$ autoantibodies in these pulmonary tuberculosis were not proved [7]. Meanwhile, remarkable high titer of anti-IFN- $\gamma$ autoantibodies found in tuberculosis is rare reported. Before our case, there was only one Thai woman with a disseminated tuberculosis single infection and a dramatic paradoxical inflammatory response after treatment initiation, was found to have a high-titer of neutralizing anti-IFN- $\gamma$ autoantibodies [4]. However, our patient had disseminated tuberculosis and a TM co-infection combined with a high level of anti-IFN- $\gamma$ autoantibodies, phenomena that triggered an inflammatory storm, resulting in rapid death. Moreover, our patient's serum could inhibit IFN $\gamma$-induced $\mathrm{CD}^{+}{ }^{+} \mathrm{T}$ cells' STAT-1 phosphorylation and Th1 cell differentiation in normal PBMCs. These observations suggest that the immunodeficiency mechanism of the anti-IFN- $\gamma$ autoantibody may include inhibition of the $\mathrm{CD}^{+}{ }^{+} \mathrm{T}$ cells' IFN- $\gamma / \mathrm{pSTAT}-1 / \mathrm{Th} 1$ pathway, ultimately leading to a severely compromised Th1 response. Thus, Th1 cells immunodeficiency due to anti-IFN- $\gamma$ autoantibodies was the cause of the severe and fatal multiple intracellular pathogen infections in this HIVnegative adult. Furthermore, the levels of proinflammatory cytokines were increased significantly in our patient, especially IL-17 A, IL-6, IL-8, TNF- $\alpha$, G-CSF, and IL-10.

Browne, et al. identified a major epitope using antiinterferon- $\gamma$ autoantibodies in patients with mycobacterial disease that showed a homology to the Aspergillus protein and found that anti-IFN- $\gamma$ autoantibodies cross-reacted with Aspergillus and Mycobacterium intracellulare Noc2 [8]. These results suggest that when a patient is infected with TM and TB, these forms of molecular mimicry may trigger the production of these autoantibodies. Thus, the anti-IFN- $\gamma$ autoantibody titer increased significantly as the condition worsened during the disease course.

In conclusion, clinicians should be aware that HIVnegative individuals with relapsing, refractory, fatal multiple intracellular pathogen infections, may have multiple immunodeficiencies, including primary immunodeficiency and/or secondary immunodeficiency, that are unrecognized. Thus, systematic genetic testing and immunological investigations should be performed for such patients.

\section{Abbreviations}

IFN-ү: Interferon-ү; PBMCs: Peripheral blood mononuclear cells; mNGS: Metagenomics next-generation sequencing; WBC: White blood cell; N: Neutrophil; ESR: Erythrocyte sedimentation rate; CRP: C-reactive protein; PCT: Procalcitonin; CT: Computerized tomography; BALF: Bronchoscopy alveolar lavage fluid; EDTA: Ethylene diamine tetraacetic acid; PBS: Phosphate buffer saline; ELISA: Enzyme-linked immunosorbent assay; MFI: Mean fluorescent intensity; PMA: Phorbol myristate acetate; DNB: DNA Nanoballs; PMDB: Pathogens Metagenomics Database; WES: Whole exome sequencing.

\section{Supplementary Information}

The online version contains supplementary material available at https://doi. org/10.1186/s12879-021-07015-5.

Additional file 1. Detailed methods for Anti-IFN- $\gamma$ autoantibody assay, Bio-Plex $^{\mathrm{TM}} 25$ cytokine assay, flow cytometry, DNA extraction, DNA libraries and sequencing, and bioinformatics analysis are provided in Additional file.

\section{Acknowledgements}

The authors thank Meng Li, Professor of Microbiology, Department of Microbiology laboratory, the First Affiliated Hospital of Guangxi Medical University.

\section{Authors' contributions}

JZ conceived of the study, critically revised the manuscript for important intellectual content. YQ conceived and designed the study, acquired, analyzed, and interpreted the data; and drafted the manuscript. MP, ZY and WZ analyzed and interpreted the data. $\mathrm{HZ}$ conceived of the study and modified the manuscript. ZL acquired and analyzed the data and agreed to be accountable for all aspects of the work for ensuring that questions related to the accuracy or integrity of any part of the work are appropriately investigated and resolved. All authors read and approved the final manuscript.

\section{Funding}

This work was supported by Grants from the Natural Science Foundation of China [NSFC81760010 and 82060364] and the Science and Technology Department of Guangxi Zhuang Autonomous Foundation of Guangxi Key Research and Development Program (No. GuikeAB20238025) from Jianquan Zhang for analysis and interpretation of data and in writing the manuscript.

\section{Availability of data and materials}

The DNA sequencing of the patient's data were submitted to the NCBI GenBank database (The GenBank accession number for the patient's nucleotide sequence: OL537177). The other data generated or analyzed during this study are included in this published article. 


\section{Declarations}

Ethics approval and consent to participate

This study was approved by the Ethical Review Committee of the First Affiliated Hospital of Guangxi Medical University (2020.KY-E-023). As the patient has died, written informed consent was obtained from the patients' daughter. Copies of the written consents are available for review.

\section{Consent for publication}

The patient's next-of-kin gave written consent for their relative's personal or clinical details along with any identifying images to be published in this study.

\section{Competing interests}

All authors declare that there are no reported conflicts of interest. All authors had access to the data and a role in writing the manuscript. All authors have read and approved the manuscript.

\section{Author details}

'Department of Respiratory and Critical Medicine, The Eighth Affiliated Hospital, Sun Yat-Sen University, Shenzhen 518000, Guangdong, China. ${ }^{2}$ Department of Comprehensive Internal Medicine, The Affiliated Tumor Hospital of Guangxi Medical University, Nanning 530021, Guangxi, China. ${ }^{3}$ Department of Respiratory and Critical Medicine, The First Affiliated Hospital of Guangxi Medical University, Nanning 530021, Guangxi, China. ${ }^{4}$ State Key Laboratory of Respiratory Disease, National Clinical Research Center for Respiratory Disease, Guangzhou Institute of Respiratory Health, The First Affiliated Hospital of Guangzhou Medical University, Guangzhou 510120, China.

Received: 25 November 2020 Accepted: 23 December 2021

Published online: 28 January 2022

\section{References}

1. Browne SK, Burbelo PD, Chetchotisakd P, et al. Adult-onset immunodeficiency in Thailand and Taiwan. N Engl J Med. 2012;367(8):725-34.

2. Zeng W, Qiu Y, Tang S, Zhang J, Pan M, Zhong X. Characterization of Anti-interferon-gamma antibodies in hiv-negative patients infected with disseminated Talaromyces marneffei and Cryptococcosis. Open Forum Infect Dis. 2019;6(10):ofz208.

3. Qiu Y, Huang J, Li Y, et al. Talaromyces marneffei and nontuberculous mycobacteria co-infection in HIV-negative patients. Sci Rep. 2021;11(1):16177.

4. Xie YL, Rosen LB, Sereti I, et al. Severe paradoxical reaction during treatment of disseminated tuberculosis in a patient with neutralizing anti-IFNY autoantibodies. Clin Infect Dis. 2016;62(6):770-3.

5. Guo J, Ning XQ, Ding JY, et al. Anti-IFN- $y$ autoantibodies underlie disseminated Talaromyces marneffei infections. J Exp Med. 2020;217(12):e20190502.

6. Qiu Y, Feng X, Zeng W, et al. Immunodeficiency disease spectrum in HIV-negative individuals with Talaromycosis. J Clin Immunol. 2021:41(1):221-3.

7. Madariaga L, Amurrio C, Martín G, et al. Detection of anti-interferongamma autoantibodies in subjects infected by Mycobacterium tuberculosis. Int J Tuberc Lung Dis. 1998;2:62-8.

8. $\mathrm{Lin} \mathrm{CH}$, Chi CY, Shih HP, et al. Identification of a major epitope by antiinterferon-gamma autoantibodies in patients with mycobacterial disease. Nat Med. 2016;22(9):994-1001.

\section{Publisher's Note}

Springer Nature remains neutral with regard to jurisdictional claims in published maps and institutional affiliations.

Ready to submit your research? Choose BMC and benefit from:

- fast, convenient online submission

- thorough peer review by experienced researchers in your field

- rapid publication on acceptance

- support for research data, including large and complex data types

- gold Open Access which fosters wider collaboration and increased citations

- maximum visibility for your research: over 100M website views per year

At BMC, research is always in progress.

Learn more biomedcentral.com/submissions 